\title{
The exterior unsteady viscous flow and heat transfer due to a porous expanding or contracting cylinder
}

\author{
Chao Wang ${ }^{\mathrm{a}}$, Xinhui Si ${ }^{\mathrm{a}, *}$, Yanan Shen ${ }^{\mathrm{a}}$, Liancun Zheng ${ }^{\mathrm{a}}$ and Ping Lin ${ }^{\mathrm{b}}$ \\ ${ }^{a}$ Department of Applied Mathematics, University of Science and Technology, Beijing, 100083, China \\ ${ }^{\mathrm{b}}$ Numerical Analysis School of Engineering, Physics and Mathematics, University of Dundee, DD1 \\ $4 H N$, Scotland
}

\begin{abstract}
Since the vessels in the biological tissues are characterized by low seepage Reynolds numbers and contracting or expanding walls, more attention is paid on the viscous flow outside the porous pipe with small expansion or contraction. This paper presents a numerical solution of the flow and heat transfer outside an expanding or contracting porous cylinder. The coupled nonlinear similarity equations are solved by Bvp4c, which is a collocation method with MATLAB. The effects of the different physical parameters, namely the permeability Reynolds number,the expansion ratio and the Prandtl number, on the velocity and temperature distribution are obtained and the results are shown graphically.
\end{abstract}

Keywords: Expanding porous cylinder, expansion ratio, similarity solution, heat transfer

\section{Introduction}

In biological organisms, many studies are made on a particular fluid transport inside porous vessel with small expansion or contraction, which help us to better understand the function of biological filters such as kidneys and lungs[1]. Uchida and Aoki [2] first proposed the model of unsteady flow in a pipe with expanding or contracting walls. In their work, the parameter of expansion ratio was introduced to describe the movement of the wall and the pipe was impermeable. Later, Goto and Uchida [3] considered the unsteady incompressible laminar flow in a semi-infinite expanding pipe with injection or suction through the porous wall. Bujurke et al.[4] analyzed the unsteady flow in a semi-infinite contracting or expanding pipe by series method. Considering the different values of Reynolds number and expansion ratio,the asymptotic and numerical solutions were also obtained by Majdalani, Zhou [5-7], Dauenhauer and Majdalani [8]. Currently the flow through the porous deforming channel was investigated by Asghar et al.[9] and Dinarvand et al.[10,11] using Adomian decomposition method(AMD) and Homotopy analysis method(HAM), respectively. The Lie symmetric method was also used by Boutros[12,13] to investigate

\footnotetext{
*Address for correspondence:Xinhui Si, Department of Applied Mathematics, University of Science and Technology, Beijing, 100083, China.Tel.:62332993; Fax:62332993; E-mail:sixinhui_ustb@126.com.
} 
the flow in pipe or channel with the same boundary conditions, respectively. Furthermore, the asymmetric laminar flow in a porous channel was discussed by Si et al.[14], in which they analyzed the effects of the different permeability Reynolds number with considering the expansion ratio. However, all of above works, the fluid considered in a channel or pipe is Newtonian fluid. Srinivasacharya et al.[15] first studied numerically the flow of a couple stress fluid in a porous channel with deforming walls using quasilinearization technique. Recently, Xu et al.[16] , Si et al.[17,18] discussed the existence of multiple solutions for the flow through porous channel or pipe with deforming walls using HAM or singular perturbation method, respectively.

Furthermore, some reports were found in literatures for the fluids outside the deforming walls. The pioneering work was done by Wang[19], who considered the flow over stretching cylinders and obtained the asymptotic solution for large Reynolds number using perturbation method. Ishak et al.[20] studied the MHD flow and heat transfer due to a stretching cylinder, and they [21] also investigated the effect of uniform suction/injection on flow and heat transfer due to a stretching cylinder. Both problems were solved numerically by the Keller-box method. Aldos and Ali [22] discussed the MHD free forced convection from a horizontal cylinder with suction and blowing. Recently, Fang et.al[23] discussed the unsteady viscous flow on the outside of an expanding or contracting cylinder and there is no permeability on the boundary.

The aim of this paper is to investigate the exterior fluid flow and heat transfer due to the orthogonally moving wall of a porous hollow tube. The exterior problem is usually difficult to be solved numerically by a direct solver for Navier-Stokes equations because of the unbounded domain. The problem can be solved by a BVP solver of ODEs. The effects of different parameters, especially the expansion ratio and the permeability Reynolds number, on the velocity fields and temperature distribution are studied and shown graphically.

\section{Formulation of the problem}

Consider the laminar flow of an incompressible viscous fluid caused by a porous cylinder, whose radius is $a(t)$ and expands or contracts uniformly at a time-dependent rate $\dot{a}(t)$. As shown in Figure 1 , the $z$-axis is measured along the axis of the cylinder and $r$-axis is measured in the radial direction. The wall has equal permeability $v_{w}$. Assume $u$ and $v$ to be the velocity components in the $z$ and $r$ directions, respectively. The surface of the cylinder is a constant temperature $T_{w}$ and the ambient fluid temperature is $T_{\infty}$, where we assume $T_{w}>T_{\infty}$. The viscous dissipation is neglected.

Under these assumptions, the governing equations can be expressed as follows[19-21,23]:

$$
\begin{array}{r}
\frac{\partial u}{\partial z}+\frac{\partial v}{\partial r}+\frac{v}{r}=0 \\
\frac{\partial u}{\partial t}+u \frac{\partial u}{\partial z}+v \frac{\partial u}{\partial r}=\nu\left(\frac{\partial^{2} u}{\partial r^{2}}+\frac{1}{r} \frac{\partial u}{\partial r}\right) \\
\frac{\partial v}{\partial t}+u \frac{\partial v}{\partial z}+v \frac{\partial v}{\partial r}=-\frac{1}{\rho} \frac{\partial p}{\partial r}+\nu\left(\frac{\partial^{2} v}{\partial r^{2}}+\frac{1}{r} \frac{\partial v}{\partial r}-\frac{v}{r^{2}}\right) \\
\frac{\partial T}{\partial t}+u \frac{\partial T}{\partial z}+v \frac{\partial T}{\partial r}=\frac{k}{\rho c}\left(\frac{\partial^{2} T}{\partial z^{2}}+\frac{\partial^{2} T}{\partial r^{2}}\right)
\end{array}
$$




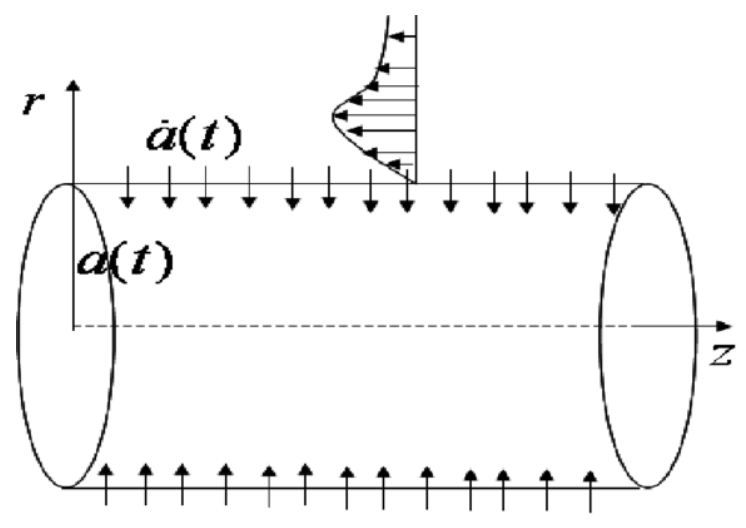

Fig. 1. porous cylinder with expanding wall

where $\rho, p, \nu, c$ and $k$ are density, pressure, kinematic viscosity, specific heat at constant pressure and the coefficient of thermal conductivity, respectively. According to Refs.[12,13,19,23], the boundary conditions are

$$
u=0, v=-v_{w}=-A \dot{a}, T=T_{w} ; r=a(t), v \rightarrow 0, T \rightarrow \infty ; r \rightarrow \infty
$$

where $A=\frac{v_{w}}{\dot{a}}$ is a constant, which is considered as the measure of wall permeability $[12,13]$. Introduce the similar transfoms[13]

$$
u=\frac{\nu z F_{\xi}(\xi, t)}{a^{2} \xi} \text { and } \psi=\nu z F(\xi, t), v=-\frac{\nu F(\xi, t)}{a \xi}, \theta=\frac{T-T_{\infty}}{T_{w}-T_{\infty}}, \eta=\xi^{2}
$$

Substituting $\psi, \theta$ into Eqs.(1)(2)(3) and (4), one obtains the following differential equations:

$$
\begin{aligned}
\eta f^{\prime \prime \prime}+f^{\prime \prime}+\frac{R e}{2}\left(f^{\prime \prime} f-f^{2}\right)+\frac{\alpha}{2}\left(\eta f^{\prime \prime}+f^{\prime}\right) & =0, \\
\eta \theta^{\prime \prime}+\frac{\theta^{\prime}}{2}(\operatorname{Pr} \eta \alpha+\operatorname{Pr} \operatorname{Re} f+1) & =0,
\end{aligned}
$$

where $R e=\frac{a v_{w}}{\nu}$ is the permeability Reynolds number, $\operatorname{Pr}=\frac{\rho c v}{k}$ is the Prandtl number, $f=\frac{F}{R e}$ and $\alpha=\frac{a \dot{a}}{\nu}$ is the wall expansion ratio. It should be noted that the expansion ratio is positive or negative depending on the expanding or contracting walls, respectively.

The corresponding boundary conditions become

$$
\begin{array}{r}
f^{\prime}(1)=0, f(1)=1, f^{\prime}(\infty)=0, \\
\theta(1)=1, \theta(\infty)=0 .
\end{array}
$$



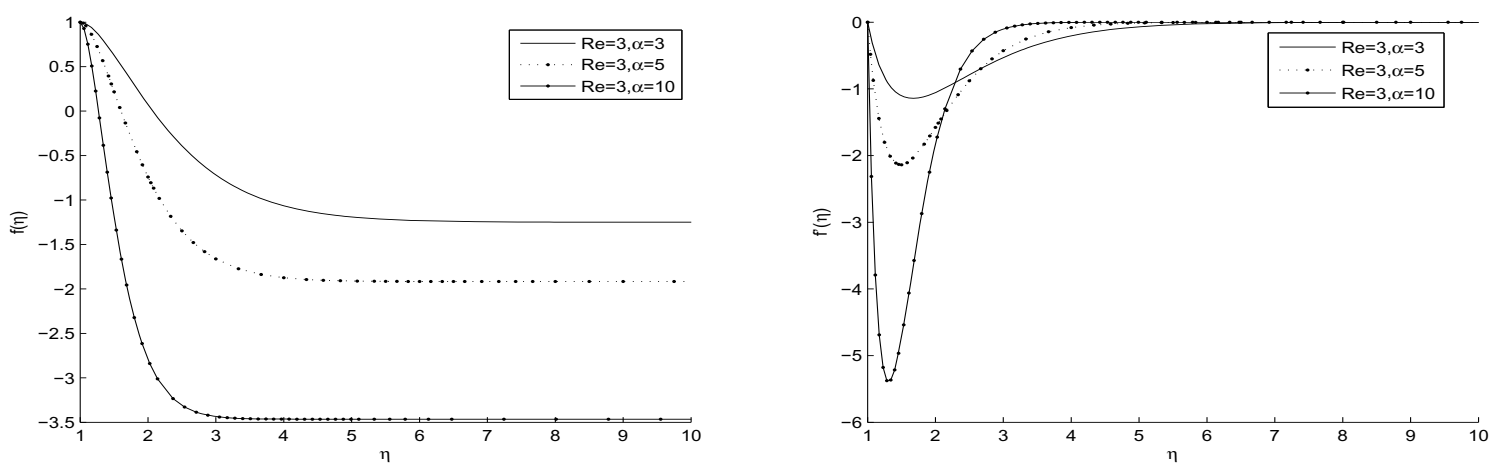

Fig. 2. variation of $f, f^{\prime}$ for different $\alpha$ as $R e=3$
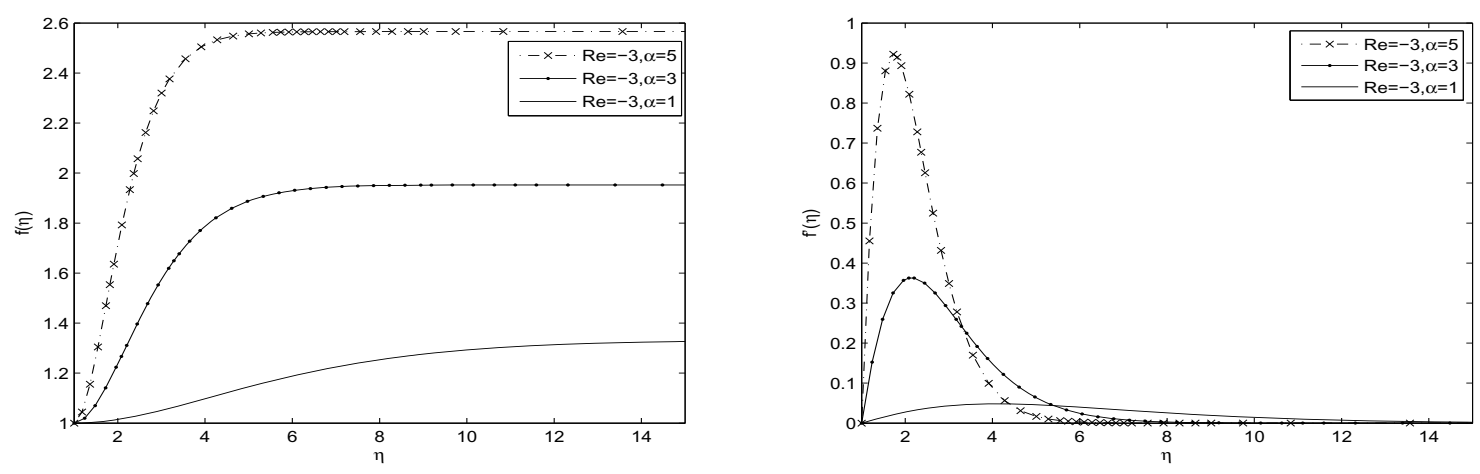

Fig. 3. variation of $f, f^{\prime}$ for different $\alpha$ as $R e=-3$

\section{Results and discussion}

Obviously, there exists a trivial solution $f(\eta)=1$, which satisfies the Eq.(7) and boundary conditions (9). The non-trivial solution is obtained numerically using a collocation method equivalent to the fourth order mono-implicit-Runge-Kutta method programmed in MATLAB[24].

Figures 2,3 show the effects of the expansion ratio on the velocity as the permeability Reynolds number is fixed. As there exists suction velocity on the wall $(R e=3)$, the radical velocity is a decreasing function of expansion ratio and the magnitude of the peak of the axial velocity also decreases with the increasing values of expansion ratio when the cylinder is expanding ( $\alpha$ is positive).In addition, when $\eta \rightarrow \infty$, the axial velocity becomes zero and the radical velocity becomes a constant. As there exists injection velocity on the wall, the profiles of the radical and axial velocity are different dramatically compared with Figure 2. The radical velocity is an increasing function of expansion ratio and the magnitude of the maximum axial velocity also increases with the expansion ratio when the cylinder is expanding ( $\alpha$ is positive). When $\eta \rightarrow \infty$, the axial velocity also becomes zero and the radical velocity becomes a constant. Figure 4 presents the temperature distribution for various values of $\alpha$. It is observed that the temperature decreases with increasing expansion ratio, which decreases with the increasing distance from the surface and finally vanishes at some large distance from the surface of the cylinder. 

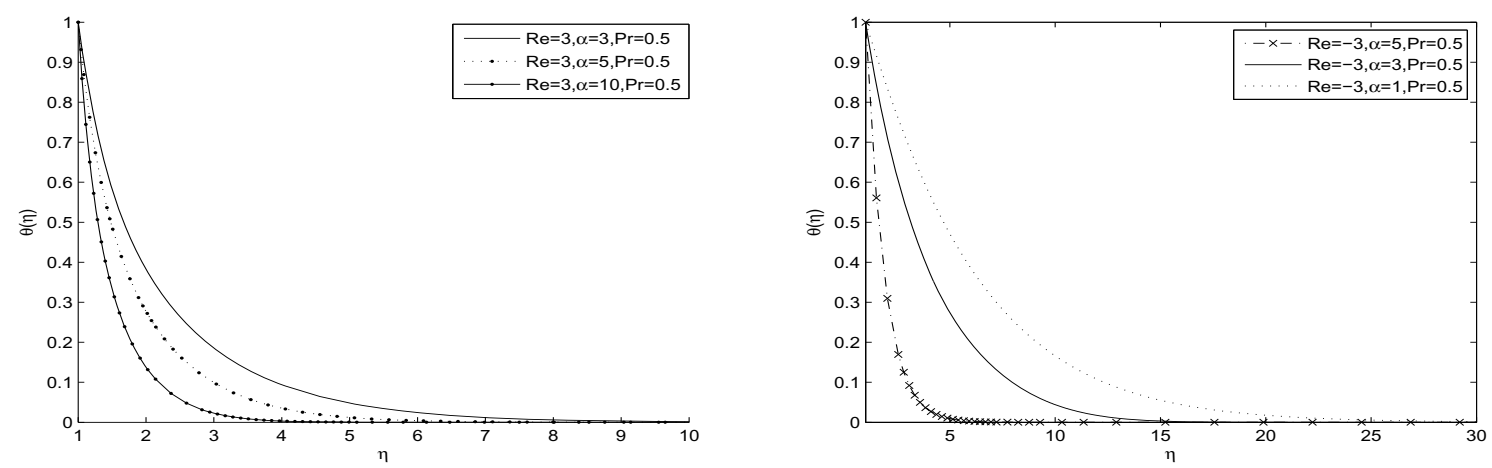

Fig. 4. variation of $\theta(\eta)$ for different $\alpha$ as $\operatorname{Pr}=0.5$
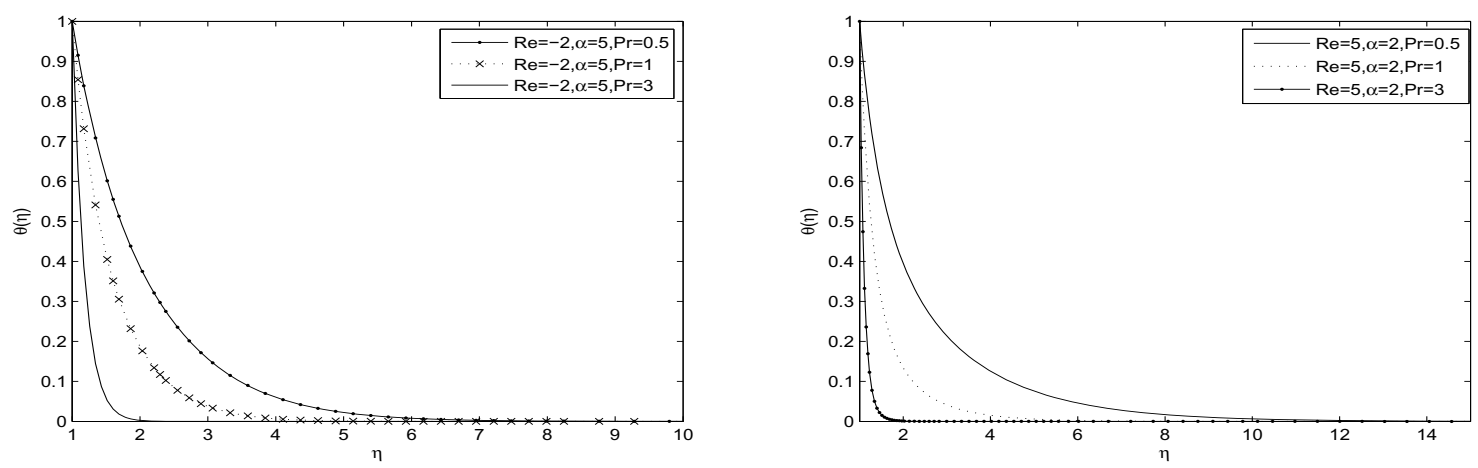

Fig. 5. variation of $\theta(\eta)$ for different $P r$

Figure 5 gives the illustration of the effects of the Prandtl number on the temperature distribution. We can find that the temperature is the decreasing function of Prandtl number, and it decreases from 1 to 0 as the distance from the surface increases.

\section{Conclusion}

The solution for the laminar flow outside a porous deforming pipe is obtained by a combined analytical/numerical method. Using suitable transformation, the Navier-Stokes equations are reduced to a system of nonlinear ordinary differential equations, which includes the expansion ratio and the Reynolds number. This boundary problem includes dual solutions, one of which is a trivial solution. The non-trivial solution is obtained by a fourth order collocation method, which is influenced by the Reynolds number and the expansion ratio. For the temperature distribution, we can found that the temperature is the decreasing function of the Prandtl number, the permeability Reynolds number and the expansion ratio. 


\section{Acknowledgments}

The work is supported by the National Natural Science Foundations of China (No.11302024), the Fundamental Research Funds for the Central Universities (Nos. FRF-BR -12-005, FRF-TP -14-071A2) and Beijing Higher Education Young Elite Teacher Project (No.YETP0387).

\section{References}

[1] H.N. Chang, J.S. Ha, J.K. Park, I.H. Kim and H.D. Shin, Velocity field of pulsatile flow in a porous tube, Journal of Biomechanics 22(1989), 1257-1262.

[2] S.Uchida, H.Aoki, Unsteady flows in a semi-infinite contracting or expanding pipe, Journal of Fluid Mechanics 82(1977), 371-387.

[3] M.Goto, S.Uchida, Unsteady flow in a semi-infinite expanding pipe with injection through wall, Japan Society for Aeronautical and Space Sciences 33(1990), 14-27

[4] N.M. Bujurke, N.P. Pai and G. Jayaraman, Computer extended series solution for unsteady flow in a contracting or expanding pipe, Ima Journal of Applied Mathematics 60(1998), 151-165.

[5] J. Majdalani and C. Zhou, Moderate-to-large injection and suction driven channel flows with expanding and contracting walls, Zeitschrift fur Angewandte Mathematik und Mechanik 83(2001)181-196.

[6] J. Majdalani and C. Zhou, Large injection and suction driven channel flows with expanding and contracting walls, 31st AIAA Fluid Dynamics Conference Anaheim, California, 2003, pp. 11-14.

[7] J. Majdalani, C. Zhou and C.A. Dawson, Two-dimensional viscous flows between slowly expanding or contracting walls with weak permeability, Journal of Biomechanics 35(2002), 1399-1403.

[8] C.E. Dauenhauer and J. Majdalani, Exact self-similarity solution of the Navier-stokes equations for a porous channel with orthogonally moving walls, Physics of Fluids 15(2003), 1485-1495.

[9] S. Asghar, M. Mushtaq and T. Hayat, Flow in a slowly deforming channel with weak permeability: an analytical approach, Nonlinear Analysis-Real World Applications 11(2010), 555-561.

[10] S. Dinarvand and M.M. Rashidi, A reliable treatment of a homotopy analysis method for two-dimensional viscous flow in a rectangular domain bounded by two moving porous walls, Nonlinear Analysis-Real World Applications 11(2010), $1502-1512$.

[11] S. Dinarvand, Viscous flow through slowly expanding or contracting porous walls with low seepage Reynolds number: a model for transport of biological fluids through vessels, Computer Methods in Biomechanics and Biomedical Engineering 14(2011), 853-862.

[12] Y.Z. Boutros, M.B. Abd-el-Malek, N.A. Badran and H.S. Hassan, Lie-group method solution for two dimensional viscous flow between slowly expanding or contracting walls with weak permeability, Applied Mathematical Modelling, 31(2007), 1092-1108.

[13] Y.Z. Boutros, M.B. Abd-el-Malek, N.A. Badran and H.S. Hassan, Lie-group method for unsteady flows in a semi-infinite expanding or contracting pipe with injection or suction through a porous wall, Journal of Computational and Applied Mathematics 197(2006), 465-494

[14] X.H. Si, L.C. Zheng, X.X. Zhang and Y. Chao, Homotopy analysis solutions for the asymmetric laminar flow in a porous channel with expanding or contracting walls, Acta Mechanica Sinica 27(2)(2011), 208-214.

[15] D. Srinivasacharya, N. Srinivasacharyulu and O. Odelu, Flow and heat transfer of couple stress fluid in a porous channel with expanding and contracting walls, International Communications in Heat and Mass Transfer, 36(2009), 180-185.

[16] H. Xu, Z.L. Lin, S.J. Liao, J.Z. Wu, J. Majdalani, Homotopy based solutions of the Navier-Stokes equations for a porous channel with orthogonally moving walls, Physics of Fluids 22(2010), 053601.

[17] X.H. Si, L.C. Zheng, X.X. Zhang and Y. Chao, The existence of multiple solutions for the laminar flow in a porous channel with suction at both slowly expanding or contracting walls, International Journal of Minerals, Metallurgy and Materials 18(2011), 494-501.

[18] X.H. Si, L.C. Zheng, X.X. Zhang, M. Li, J.H. Yang and Y. Chao, Multiple solutions for the laminar flow in a porous pipe with suction at slowly expanding or contracting wall, Applied Mathematics and Computation 218(2011), 3515-3521.

[19] C.Y. Wang, Fluid flow due to a stretching cylinder, Physics of Fluids 31(1988), 466-468.

[20] A. Ishak, R. Nazar and I. Pop, Magnetohydrodynamic (MHD) flow and heat transfer due to a stretching cylinder, Energy Conversion and Management, 49(2008), 3265-3269.

[21] A. Ishak, R. Nazar and I. Pop, Uniform suction/blowing effect on flow and heat transfer due to a stretching cylinder, Applied Mathematical Modelling, 32(2008), 2059-2066.

[22] T.K. Aldos and Y.D. Ali, MHD free forced convection from a horizontal cylinder with suction and blowing, International Communications in Heat and Mass Transfer 24(1997), 683-693. 
[23] T.G. Fang, J. Zhang and Y.F. Zhong, Note on unsteady viscous flow on the outside of an expanding or contracting cylinder, Communications in Nonlinear Science and Numerical Simulation 2011(in press).

[24] J. Kierzenka and L.F. Shampine, A BVP solver based on residual control and the MATLAB PSE, Transactions on Mathematical Software 27(2001), 299-316. 